Original Article

\title{
Decursin induces apoptosis in glioblastoma cells, but not in glial cells via a mitochondria-related caspase pathway
}

\author{
Seung Tack Oh', Seongmi Lee², Cai Hua ${ }^{3}$, Byung-Soo Koo ${ }^{4}$, Sok Cheon Pak ${ }^{5}$, Dong-II-Kim ${ }^{6, *}$, Songhee Jeon ${ }^{3, *}$, and \\ Boo Ahn Shin ${ }^{7, *}$
}

${ }^{1}$ Research Institute, Dongkwang Pharmaceutical Company, Ltd., Seoul 04535, Korea, ${ }^{2}$ Department of Child and Adolescent Psychiatry, National Center for Mental Health, Seoul 04933, Korea, ${ }^{3}$ Department of Biomedical Sciences, Center for Creative Biomedical Scientists at Chonnam National University, Gwangju 61469, Korea, ${ }^{4}$ Department of Neuropsychiatry, College of Korean Medicine, Dongguk University, Goyang 10326, Korea, ${ }^{5}$ School of Biomedical Sciences, Charles Sturt University, Bathurst, NSW 2795 Australia, ${ }^{6}$ Department of Obstetrics \& Gynecology, College of Korean Medicine, Dongguk University, Goyang 10326, Korea, ${ }^{7}$ Department of Microbiology and Immunology, Chonnam National University Medical School, Gwangju 61469, Korea

\section{ARTICLE INFO}

Received January 9, 2017

Revised October 10, 2018

Accepted November 6, 2018

*Correspondence

Dong-II Kim

E-mail: obgykdi@hanmail.net

Songhee Jeon

E-mail: jsong0304@jnu.ac.kr

Boo Ahn Shin

E-mail: bashin@jnu.ac.kr

Key Words

Anti-cancer activity

Apoptosis

Cell cycle arrest

Decursin

Glioblastoma
ABSTRACT Decursin is a major biological active component of Angelica gigas Nakai and is known to induce apoptosis of metastatic prostatic cancer cells. Recently, other reports have been commissioned to examine the anticancer activities of this plant. In this study, we evaluated the inhibitory activity and related mechanism of action of decursin against glioblastoma cell line. Decursin demonstrated cytotoxic effects on U87 and C6 glioma cells in a dose-dependent manner but not in primary glial cells. Additionally, decursin increased apoptotic bodies and phosphorylated JNK and p38 in U87 cells. Decursin also down-regulated $\mathrm{Bcl}-2$ as well as cell cycle dependent proteins, CDK-4 and cyclin D1. Furthermore, decursin-induced apoptosis was dependent on the caspase activation in U87 cells. Taken together, our data provide the evidence that decursin induces apoptosis in glioblastoma cells, making it a potential candidate as a chemotherapeutic drug against brain tumor.

\section{INTRODUCTION}

Angelica gigas (Dang Gui) is a monocarpic biennial plant from China, Korea and Japan, and is used in traditional herbal medicine. It inhabits forests, grasslands and banks of streams. The dried roots are used in the treatment of female reproductive health issues such as dysmenorrhea, amenorrhea and menopausal syndromes by enhancing blood circulation and new blood synthesis [1].

Decursin, a coumarin derivative, is one of major constituents of this plant [2] and has been reported to inhibit the growth and survival of some metastatic prostatic cancer cells $[3,4]$. However, there have been no studies conducted on the use of decursin for the prevention or treatment of brain tumors. The most common primary central nervous system tumor, glioblastoma, represents about $30 \%$ of all brain tumors and $80 \%$ of all malignant tumors. It can be categorized into four grades (I to IV), where grades I and II reflect low-grade gliomas with grades III and IV (glioblastoma) being high-grade gliomas. Nearly $60 \%$ of high-grade gliomas are glioblastoma, and the incidence rate for these tumors is approximately 3 per 100,000 [5]. The current clinically used treatment strategies for glioblastoma include surgery followed by concurrent
This is an Open Access article distributed under the terms of the Creative Commons Attribution Non-Commercial License, which permits unrestricted non-commercial use, distribution, and reproduction in any medium, provided the original work is properly cited. Copyright @ Korean J Physiol Pharmacol, pISSN 1226-4512, elSSN 2093-3827
Author contributions: S.T.O. and S.M.L. carried out cell experiments and western blotting and C.H. carried out primary glial cell culture. B.S.K., S.C.P. and D.I.K. wrote the draft manuscript. S.H.J. and B.A.S. organized the study. 
ionizing radiation and chemotherapy. Glioblastoma patients have poor prognosis with a median survival time of only approximately one year due to the rapid proliferation and accelerated activities of tumor cells. Unfortunately, the 5 -year survival rate is only $9.8 \%$ [6]. Decursin-mediated glioblastoma treatment has rarely been studied. Thus, the purpose of this study was to evaluate the effects of decursin on glioblastoma using a human glioblastoma cell line, U87 and rat glioma cell line, C6.

\section{METHODS}

\section{Cell culture}

A human glioblastoma cell line, U87 and rat glioblastoma cell line, C6 were purchased from the American Type Culture Collection (Rockville, MD, USA). Cells were maintained in Dulbecco's Modified Eagle's Medium (DMEM) supplemented with 10\% fetal bovine serum (FBS) (Welgene, Seoul, Korea), $100 \mathrm{U} / \mathrm{ml}$ penicillin and $0.1 \mathrm{mg} / \mathrm{ml}$ streptomycin (Welgene, Seoul, Korea) in condition of $95 \%$ air and $5 \% \mathrm{CO}_{2}$ at $37^{\circ} \mathrm{C}$.

\section{Mixed glial cell culture}

Primary mixed glial cells were prepared from postnatal day 2 pups of mouse. Briefly, cerebral cortices were subjected to Trypsin-EDTA mix digestion (Invitrogen, CA, USA) for $30 \mathrm{~min}$ at $37^{\circ} \mathrm{C}$. The digested tissue was carefully triturated into single cells using increasingly smaller pipette tips. Cells were then centrifuged at $250 \times \mathrm{g}$ for $5 \mathrm{~min}$ and re-suspended in plating medium supplemented with DFF10 (DMEM:F12 1:1 + 10\% FBS) including $100 \mathrm{U} / \mathrm{ml}$ streptomycin and $100 \mu \mathrm{g} / \mathrm{ml}$ penicillin. Dissociated cells were seeded into a poly-L-lysine coated 6-well plate and incubated at $37^{\circ} \mathrm{C}$.

\section{Cell viability}

Cells were seeded into a 24 -well plate at a density of $1 \times 10^{5} / \mathrm{ml}$. The next day, cells were treated with various concentration of decursin $(10,20,50,100$ and $200 \mu \mathrm{M})$ for $24 \mathrm{~h}$ or $48 \mathrm{~h}$ incubation. MTT solution was treated for $1 \mathrm{~h}$ at $37^{\circ} \mathrm{C}$. Then, dimethyl sulfoxide was added to dissolve formazan crystals and incubated for $30 \mathrm{~min}$. The optical density of solubilized formazan crystals was measured by using a spectrophotometer (Molecular Device, Sunnyvale, CA, USA) at $580 \mathrm{~nm}$. The half maximal inhibitory concentration $\left(\mathrm{IC}_{50}\right)$ was calculated using Calcusyn software (Biosoft, Cambridge, UK). Caspase apoptosis dependency was analyzed using $50 \mu \mathrm{M}$ of pan caspase inhibitor (Cas I), p38 inhibitor (SB203580) and JNK inhibitor (SP600125) which were acquired from Calbiochem (EMD Millipore, Darmstadt, Germany). Cells were pre-treated for $30 \mathrm{~min}$ prior to treatment of 25 or $50 \mu \mathrm{M}$ of decursin. At $24 \mathrm{~h}$, cell viability was examined by MTT assy.

\section{Immunoblotting}

The cells were lysed with $1 x$ lysis buffer (2\% SDS, $1 \%$ 2-mercaptoethanol, $10 \%$ glycerol and $0.1 \mathrm{mg} / \mathrm{ml}$ bromophenol blue in Tris$\mathrm{HCl}, \mathrm{pH} 6.8$ ) and heated at $100^{\circ} \mathrm{C}$ for $10 \mathrm{~min}$. Thirty $\mu \mathrm{g}$ of cell lysates were electrophoresed in $10 \%$ or $15 \%$ SDS-PAGE and gels were transferred on nitrocellulose membrane. Then, the membrane was incubated with anti-Bcl-2, anti-Bax, anti-phosphop38, anti-p38 anti-phospho-JNK, anti-JNK, anti-caspase-3, anticleaved caspase-3, anti-caspase-7, anti-cleaved caspase-7, anticaspase-9, anti-cleaved caspase-9, anti-cleaved PARP-1, antiCDK-4, anti-CyclinD1 and anti- $\beta$ actin antibody (Cell Signaling Technology, Beverly, MA, USA) for overnight at $4^{\circ} \mathrm{C}$. To obtain band image, horseradish peroxidase-conjugated anti-mouse or anti-rabbit IgG was incubated on the blot for $1 \mathrm{~h}$ at room temperature. After washing the blot, bands were visualized using the ECL system (Thermo Fisher Scientific, Waltham, MA, USA). Band images were obtained by using Molecular Imager ChemiDoc XRS+ (Bio-Rad, Hercules, CA, USA) and band intensity was measured by Image $\mathrm{Lab}^{\mathrm{TM}}$ software version 2.0.1 (Bio-Rad).

\section{Annexin V}

U87 cells $\left(1 \times 10^{5} / \mathrm{ml}\right)$ were treated with decursin $(50$ or $100 \mu \mathrm{M})$ for $24 \mathrm{~h}$. Then, cells were trypsinized and re-suspended with 500 $\mu \mathrm{l}$ of $1 \mathrm{x}$ binding buffer. Apoptotic cells were calculated by double staining with Muse ${ }^{\mathrm{TM}}$ Annexin V \& Dead Cell reagent (Merck KGaA, Darmstadt, Germany) and examined by using the Muse ${ }^{\mathrm{TM}}$ Cell Analyzer (Merck KGaA, Darmstadt, Germany).

\section{Cell cycle analysis}

U87 cells were harvested, fixed in ethanol, and stored at $-20^{\circ} \mathrm{C}$. Muse $^{\mathrm{TM}}$ Cell Cycle Reagent (Merck KGaA, Darmstadt, Germany) was added in the ethanol-fixed cells and cell cycle population was acquired by using the Muse ${ }^{\mathrm{TM}}$ Cell Cycle software module (Merck KGaA, Darmstadt, Germany).

\section{Data analysis}

All statistical analyses were conducted with SPSS (ver. 21, Somers, NY, USA). The data expressed as mean \pm SD were analyzed by one-way ANOVA, and the dissimilarity among means were analyzed using the Dunnett's test or Tukey-Kramer's multiple comparison test. Differences were considered significant at $\mathrm{p}<0.05$. 


\section{RESULTS}

\section{Decursin at high doses has a cytotoxic effect in U87 cells}

To examine cytotoxic effect of decursin in U87 cells, human glioblastoma cell lines were treated with various concentrations (0-200 $\mu \mathrm{M})$ of decursin for $24 \mathrm{~h}$ and then analyzed by MTT assay. As shown in Fig. 1, cell viability was gradually decreased by 100 $\mu \mathrm{M}$ of decursin treatment after $24 \mathrm{~h}$, but primary glia cells were not changed by $100 \mu \mathrm{M}$. U87 glioblastoma cells almost died when treated with $200 \mu \mathrm{M}$ of decursin, but primary glial cells showed a survival rate of more than $50 \%$ (Fig. 1B), implying that high concentration of decursin showed cytotoxic effect on the normal cells. The half maximal inhibitory concentration $\left(\mathrm{IC}_{50}\right)$ value for decursin was calculated and it was $49.01 \mu \mathrm{M}$ in U87 cells. Thus, in the present study, we used $50 \mu \mathrm{M}$ to examine the mechanisms of action of decursin in the U87 cells.

\section{Decursin induces apoptosis in U87 cells}

The morphological characteristics of apoptosis were captured at $24 \mathrm{~h}$ after decursin treatment in U87 cells. As shown in Fig. 2A, decursin induced chromatin condensation and apoptotic bodies. To evaluate the potential apoptotic ability of decursin in U87 cells, we stained the cells with Annexin V and PI. At a dose of $50 \mu \mathrm{M}$, decursin induced apoptosis by $44.4 \%$ (Fig. 2B). To examine which signaling pathway may be involved in apoptosis, Western blotting was utilized in the decursin-treated U87 cells. Activations of cJun NH2-terminal kinase (JNK) and p38 were examined by using phospho-specific antibodies. Increased phosphorylation of P38 and JNK was observed from $0.5 \mathrm{~h}$ until $1 \mathrm{~h}$ of decursin treatment (Fig. 3A). We also examined the expression ratio of $\mathrm{Bcl}-2 / \mathrm{Bax}$ in U87 cells after decursin treatment. Bcl-2 inhibits apoptosis by preventing mitochondrial membrane depolarization, whereas Bax promotes apoptosis by enhancing mitochondrial membrane depolarization and cytochrome $\mathrm{c}$ release. As shown in Fig. 3B, Bcl-2 expression was decreased by treatment of 50 or $100 \mu \mathrm{M}$ decursin, but Bax expression was not changed. Moreover, the ratio of Bcl-2/ Bax was significantly decreased by decursin. Next, we examined the caspase activity and PARP-1 cleavage after 50 or $100 \mu \mathrm{M}$ of decursin treatment. Cleaved caspase-3, -7 , or -9 activities were increased by decursin treatment in a dose-dependent manner and cleaved PARP-1 also increased by $100 \mu \mathrm{M}$ of decursin, suggesting
A.

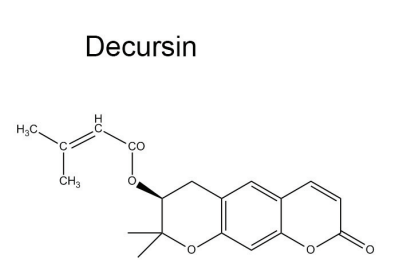

B.

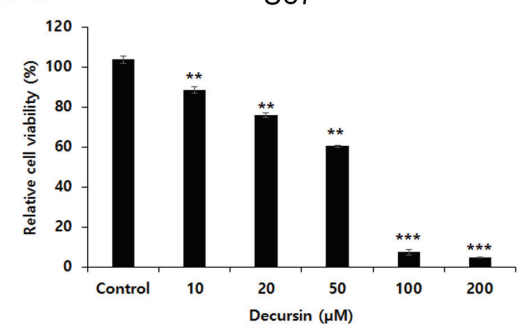

C.

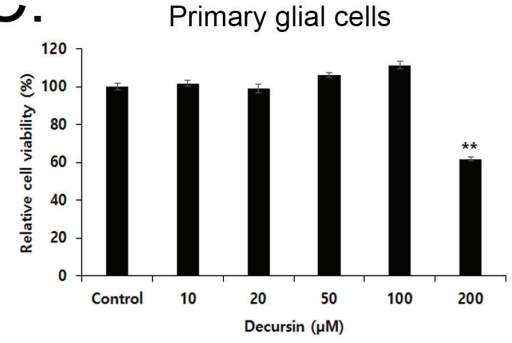

Fig. 1. Decursin has a cytotoxic effect in U87 cells but not in primary glia cells. (A) Chemical structure of decursin. (B) U87 cells were treated with various concentrations of decursin $(10,20,50,100$ and $200 \mu \mathrm{M})$ for $24 \mathrm{~h}$. (C) Primary glia cells were treated with different concentrations of decursin (10, 20, 50, 100 and $200 \mu \mathrm{M})$ for $24 \mathrm{~h}$. Cell viability was determined by the MTT assay. Values are mean $\pm S D(n=3) .{ }^{* *} p<0.01$ vs. control, and ${ }^{* * *} p<0.001$ vs. control.
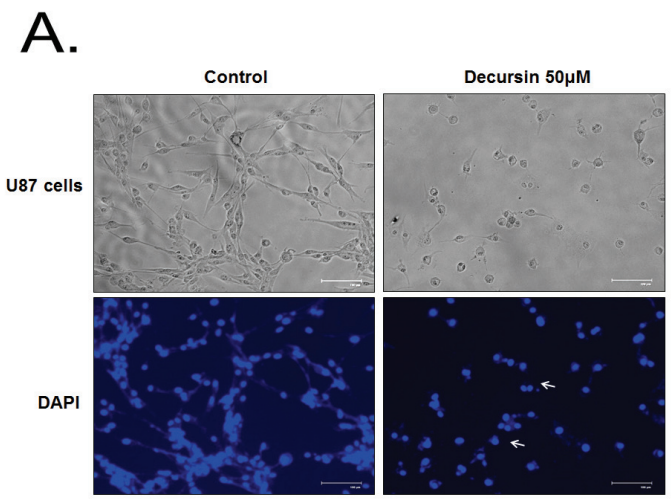

B.
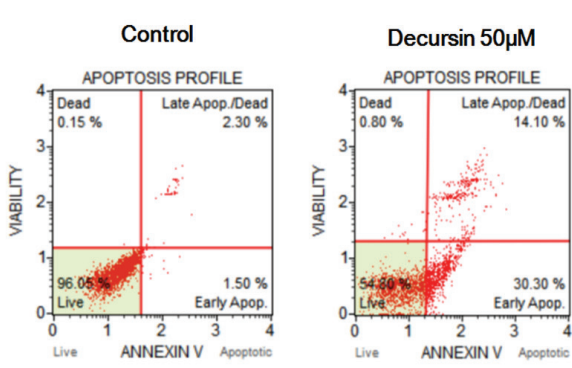

Fig. 2. Decursin induces apoptosis in U87 cells. (A) The image shows that decursin induced chromatin condensation and apoptotic bodies. (B) U87 cells were treated with $50 \mu \mathrm{M}$ of decursin for $24 \mathrm{~h}$, stained with Annexin V and PI, and analyzed with FACS system. 
A.

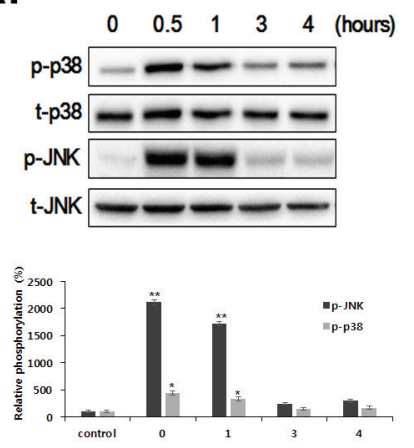

B.
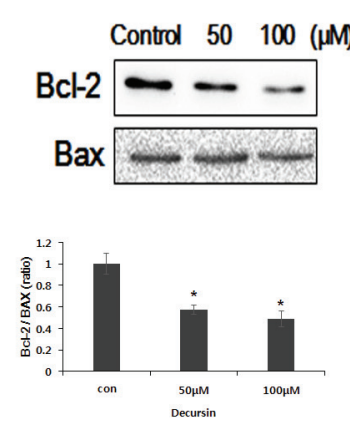

C

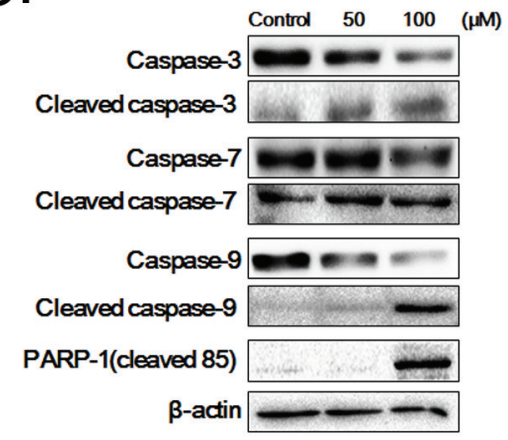

Fig. 3. Decursin induces phosphorylation of JNK and p38 in U87 cells, alters Bcl-2 family protein levels, and activates caspases and PARP-1 in U87 cells. (A) U87 cells were treated with $50 \mu \mathrm{M}$ of decursin for various periods of time $(0,0.5,1,3$ and $4 \mathrm{~h})$. The cell lysates were subjected to Western blot analysis for phospho-JNK, total-JNK, phospho-p38 and total p38. (B) U87 cells were treated with 50 or $100 \mu \mathrm{M}$ of decursin for $24 \mathrm{~h}$. The cell lysates were subjected to Western blot analysis for Bax and Bcl-2 (C), cleaved caspase-3, 7, 9, PARP-1 and $\beta$-actin. Values are mean \pm SD $(n=3)$. ${ }^{*} p<0.05$ vs. control. ${ }^{* *} p<0.01$ vs. control.

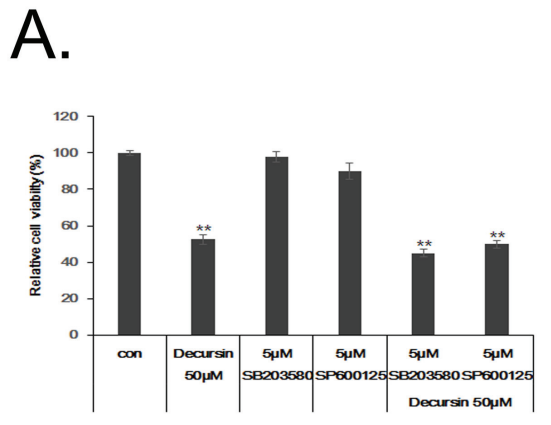

B.

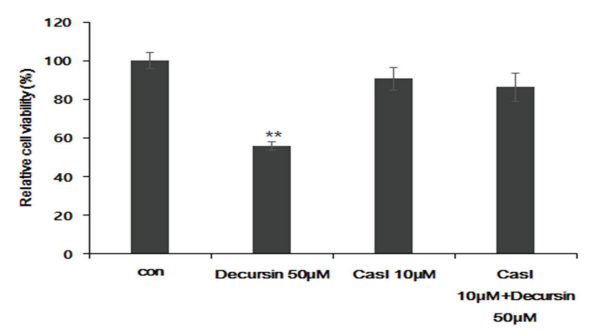

Fig. 4. Decursin induces apoptosis in a caspase-dependent manner in U87 cells. (A) U87 cells were pre-treated with $5 \mu \mathrm{M}$ of SB203580 (p38 inhibitor) and SP600125 (JNK inhibitor) for 30 min prior to decursin treatment ( $50 \mu \mathrm{M})$. Cell viability was determined by the MTT assay. (B) U87 cells were pre-treated with $10 \mu \mathrm{M}$ of pan caspase inhibitor (Cas I) for $30 \mathrm{~min}$ prior to decursin treatment ( $50 \mu \mathrm{M})$. Cell viability was determined by the MTT assay. Values are mean $\pm S D(n=4) .{ }^{* *} p<0.01$ vs. control.

that decursin induced apoptotic cell death in U87 cells (Fig. 3C).

\section{Decursin induces apoptosis by caspase-dependent manner in U87 cells}

To examine the signaling pathway responsible for the cell death, JNK or p38 kinase inhibitor was pre-treated in the decursin-treated cells. Either SB203580 as p38 inhibitor or SP600125 as JNK inhibitor did not suppress decursin-induced U87 cell death (Fig. 4A). In order to verify the importance of caspase activation in the decursin-induced apoptosis, U87 cells were pre-treated with a pan caspase inhibitor (Cas I) for 30 min prior to treatment with $50 \mu \mathrm{M}$ of decursin. At $24 \mathrm{~h}$, cell viability was examined by MTT assay. Pre-treatment with a pan caspase inhibitor significantly suppressed decursin-induced cell death in U87 cells, indicating that decursin-induced cell death is mediated by caspase activation in U87 cells (Fig. 4B).

\section{Decursin increases $\mathrm{G} 1$ population apoptosis by caspase-dependent manner in U87 cells}

Next, we analyzed cell cycle by using flow cytometry analysis. Decursin increased the amount of sub-G1 DNA population dosedependently in U87 cells compared with that of control (Fig. 5A). In order to confirm the cell cycle arrest by decursin treatment, we examined the expression level of cell cycle-dependent proteins, cyclin dependent protein kinase-4 (CDK-4) and cyclin D1, in decursin-treated U87 cells. The expression of both proteins was decreased by $100 \mu \mathrm{M}$ of decursin in U87 cells (Fig. 5), which indicates a cell cycle arrest in $\mathrm{Gl} / \mathrm{S}$ stage.

\section{Decursin induces apoptosis in C6 cells}

To confirm the cytotoxic effect of decursin on other glioma cell, rat glioma $\mathrm{C} 6$ cells were treated with various concentrations of decursin. After 24 or $48 \mathrm{~h}$, cell viability was examined by MTT method. Decursin induced cell death and caspase activation 
A.

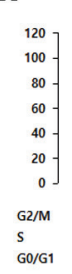

Cell cycle
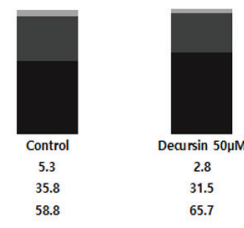

- G0/G1 $=S=G 2 / M$

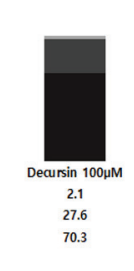

B.
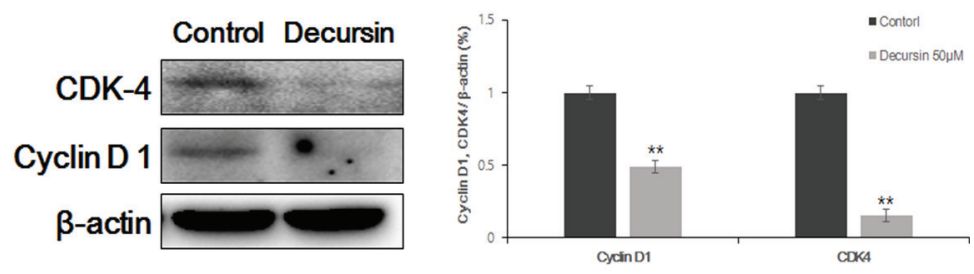

Fig. 5. Decursin increases $\mathrm{G} 1$ population of cell cycle and down-regulates cell cycle proteins in U87 cells. U87 cells were treated with $50 \mu \mathrm{M}$ of decursin for $24 \mathrm{~h}$. The cell lysates were subjected to flow cytometric analysis and Western blot analysis to examine the expression of CDK-4, cyclin D1 and $\beta$-actin. Percentage of each cell population was presented in graph (A). The intensity of the each band was normalized to $\beta$-actin and presented in bar graphs (B). Values are mean $\pm S D(n=3) .{ }^{* *} p<0.01$ vs. control.

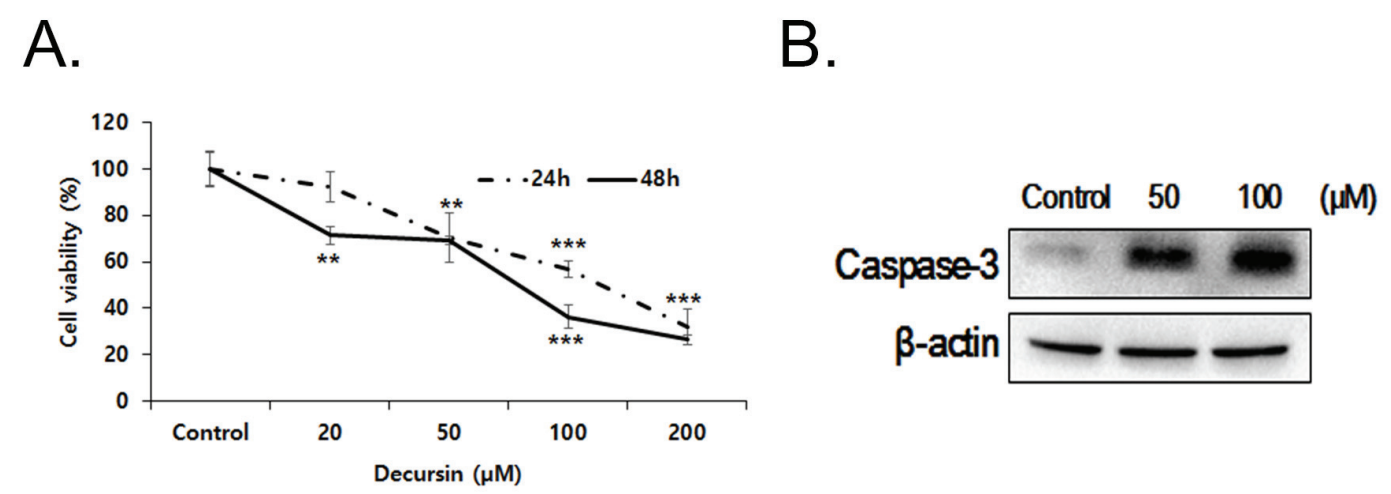

Fig. 6. Decursin induces apoptosis and caspase-3 activation in C6 cells. (A) C6 cells were treated with various concentrations of decursin (10, 20, $50,100$ and $200 \mu \mathrm{M})$ for $24 \mathrm{~h}$ and $48 \mathrm{~h}$. Values are mean $\pm \mathrm{SD}(\mathrm{n}=4)$. ${ }^{* *} \mathrm{p}<0.01 \mathrm{vs}$. control, ${ }^{* * *} \mathrm{p}<0.001 \mathrm{vs}$. control. (B) The cell lysates were subjected to Western blot analysis for cleaved caspase- 3 and $\beta$-actin.

dose-dependently in C6 cells (Fig. 6), suggesting decursin induces apoptotic cell death in glioma C6 cells.

\section{DISCUSSION}

Previous studies have shown that decursin induced apoptosis in several cancer cell lines such as prostate cancer, myeloid leukemia, breast cancer and colon cancer [7-9], however there have been no reports of the effect that decursin may have on brain tumor cells. In this study, we examined the anticancer effect of decursin on glioblastoma cell lines, U87 and C6. We showed that decursin induced apoptosis of U87 and C6 cells in a dose-dependent manner but not in primary glial cells. The effect of decursin on primary glial cells is in accordance with the previous report that decursin has neuroprotective effect in the glutamate-injured cortical cells [10].

Apoptosis is a mechanism of regulated cell death employed during normal development [11] where mitochondrial apoptotic pathways act as important signals of apoptotic cell death in mammalian cells [12]. The Bcl-2 family proteins are the major regulators of apoptosis and consist of three subfamilies such as Bcl-
2, Bax and Bik. While Bcl-2 is an anti-apoptotic member of the family, Bax is a pro-apoptotic member, and it is thought that the ratio between anti-apoptotic and pro-apoptotic members of this family may contribute to the vulnerability of a cell to apoptosis [13]. In this study, we demonstrated that decursin decreased Bcl2 expression without any change to Bax expression in U87 cells, which resulted in a significant decrease in the Bcl-2/Bax ratio and therefore may be involved in the apoptosis process.

In addition, decursin caused PARP cleavage. A decrease in the $\mathrm{Bcl}-2 / \mathrm{Bax}$ ratio may have resulted in the release of cytochrome $\mathrm{c}$ and the initiation of apoptosis. After its release, cytochrome $\mathrm{c}$ interacts with procaspase- 9 and apoptotic protease activating factor-1 (Apaf-1) to activate caspase-9, and caspase- 9 instigates a cascade of further caspase activation by processing caspases- 3 and -7 , which leads to PARP cleavage and eventually apoptosis $[14,15]$. Caspase-3 is the most well-known effector caspase and its activation leads to cellular death by the proteolytic cleavage of a large variety of cellular proteins and the activation of pro-apoptotic factors that destroy the cell $[16,17]$. Moreover, PARP cleavage during caspase activation facilitates cellular disassembly and serves as a marker of cells undergoing apoptosis [18]. This PARP cleavage was observed in U87 cells treated with decursin at concentra- 
tions of 50 and $100 \mu \mathrm{M}$, suggesting that decursin activates this mitochondria apoptotic pathway in neuroblastoma cells.

Furthermore, it has been reported that the JNK and p38 kinase pathways are related with an increased rate of apoptosis, whereas the ERK1/2 pathway activated by cell growth has been shown to be important for cell survival by inhibiting apoptosis [19-21]. However, alternate studies have demonstrated that the JNK pathway, when activated, is not involved in apoptosis of neuronal cells [22]. Other studies showed that JNK is also important for the development and survival of macrophages [23]. Furthermore, where UV and S-nitrosoglutathione were administered, p38 inhibition was unable to prevent apoptosis [24,25]. This is consistent with this study, where inhibition p38 or JNK did not block decursininduced apoptosis. These differences suggest the existence of marked alterations in the regulation of stress reactions depending on the type of cell being examined, so that the implication of either JNK or p38 is difficult to explain and may be confounded by many factors.

Cancer is unchecked cell growth. Mutations in genes can cause cancer by accelerating cell division rates or inhibiting normal controls on the system, such as cell cycle arrest or programmed cell death. Glioma cells are distinguished from normal glial cells by abnormal proliferation and anti-apoptosis. In this study, we found that decursin markedly inhibited the viabilities of U87 and C6 cells and increased the apoptosis of glioma cells in a dose-dependent manner. Besides, decursin down-regulated cyclin D1 and CDK-4 expression, which are involved in the arrest of G1 in the prostate cancer [4]. This is in concert with previous reports that decursin induced G1 arrest and apoptosis in the human prostate, bladder and colon cancer cells [26]. Thus, it is suggested that the decursin-induced G1 cell cycle arrest is through a CDK4/cyclin D1-mediated pathway in the rapidly proliferating cells, resulting in cell death of glioma cells but not primary glial cells.

In this study, $\mathrm{IC}_{50}$ of decursin was $49.01 \mu \mathrm{M}$ in U87 cells. However, the development of a natural substance as a new drug has the potential to become an $\mathrm{IC}_{50}$ in $\mathrm{nM}$ units. Indeed, decursin has an anticancer effect over $50 \mu \mathrm{M}$ in the various cancer cell line [4,26-29] and many studies have shown that decursin has synergetic effects with doxorubicin or c-myc inhibitor [27-29]. Combination therapy has been widely used as a method for overcoming the limitations of chemotherapy in the treatment of cancer [30]. Thus, decursin can be used in combination with other anticancer drugs to treat glioblastoma.

Taken together, decursin inhibited proliferation of neuroblastoma cell line via G1/S cell cycle arrest and induction of apoptosis through caspase-3 activation, and down-regulation of Bcl-2 protein expression without negative effect on primary glia cells. Malignant gliomas are the most common type of primary brain tumor that even after aggressive therapy the survival rate is poor, and thus there is an urgent need to develop specific and effective therapies [31]. Moreover, intraperitoneal injection of decursin strongly inhibited selective neuronal death, astrogliosis, and oxi- dative stress induced by KA administration [32], suggesting that decursin could penetrate brain blood barrier. Hence, decursin could be developed as an antitumor drug to treat glioblastoma patients without damage on normal brain cells.

\section{ACKNOWLEDGEMENTS}

This work was supported by a grant of the Korean Health Technology R\&D Project, Ministry of Health and Welfare, Republic of Korea (No. HN12C0062, HI15C0198).

\section{CONFLICTS OF INTEREST}

The authors declare no conflicts of interest.

\section{REFERENCES}

1. Heo J. DONGUIBOGAM Treasured Mirror of Eastern Medicine Part I Internal Bodily Elements. Seoul: Chin Young; 2013.

2. Ahn MJ, Lee MK, Kim YC, Sung SH. The simultaneous determination of coumarins in Angelica gigas root by high performance liquid chromatography-diode array detector coupled with electrospray ionization/mass spectrometry. J Pharm Biomed Anal. 2008;46:258266.

3. Rhim JS. In vitro human cell culture models for the study of prostate cancer. Prostate Cancer Prostatic Dis. 2000;3:229-235.

4. Yim D, Singh RP, Agarwal C, Lee S, Chi H, Agarwal R. A novel anticancer agent, decursin, induces G1 arrest and apoptosis in human prostate carcinoma cells. Cancer Res. 2005;65:1035-1044.

5. de Groot JF. High-grade gliomas. Continuum (Minneap Minn). 2015;21:332-344.

6. Stupp R, Mason WP, van den Bent MJ, Weller M, Fisher B, Taphoorn MJ, Belanger K, Brandes AA, Marosi C, Bogdahn U, Curschmann J, Janzer RC, Ludwin SK, Gorlia T, Allgeier A, Lacombe D, Cairncross JG, Eisenhauer E, Mirimanoff RO; European Organisation for Research and Treatment of Cancer Brain Tumor and Radiotherapy Groups; National Cancer Institute of Canada Clinical Trials Group. Radiotherapy plus concomitant and adjuvant temozolomide for glioblastoma. N Engl J Med. 2005;352:987-996.

7. Jiang C, Guo J, Wang Z, Xiao B, Lee HJ, Lee EO, Kim SH, Lu J. Decursin and decursinol angelate inhibit estrogen-stimulated and estrogen-independent growth and survival of breast cancer cells. Breast Cancer Res. 2007;9:R77.

8. Ahn Q, Jeong SJ, Lee HJ, Kwon HY, Han I, Kim HS, Lee HJ, Lee EO, Ahn KS, Jung MH, Zhu S, Chen CY, Kim SH. Inhibition of cyclooxygenase-2-dependent survivin mediates decursin-induced apoptosis in human KBM-5 myeloid leukemia cells. Cancer Lett. 2010;298:212-221.

9. Son SH, Park KK, Park SK, Kim YC, Kim YS, Lee SK, Chung WY. Decursin and decursinol from Angelica gigas inhibit the lung metastasis of murine colon carcinoma. Phytother Res. 2011;25:959-964.

10. Kang SY, Kim YC. Decursinol and decursin protect primary cul- 
tured rat cortical cells from glutamate-induced neurotoxicity. $J$ Pharm Pharmacol. 2007;59:863-870.

11. Waxman DJ, Schwartz PS. Harnessing apoptosis for improved anticancer gene therapy. Cancer Res. 2003;63:8563-8572.

12. Chen M, Li B, Zhao X, Zuo H, He X, Li Z, Liu X, Chen L. Effect of diallyl trisulfide derivatives on the induction of apoptosis in human prostate cancer PC-3 cells. Mol Cell Biochem. 2012;363:75-84.

13. Niture SK, Jaiswal AK. INrf2 (Keap1) targets Bcl-2 degradation and controls cellular apoptosis. Cell Death Differ. 2011;18:439-451.

14. Susin SA, Daugas E, Ravagnan L, Samejima K, Zamzami N, Loeffler M, Costantini P, Ferri KF, Irinopoulou T, Prévost MC, Brothers G, Mak TW, Penninger J, Earnshaw WC, Kroemer G. Two distinct pathways leading to nuclear apoptosis. J Exp Med. 2000;192:571580.

15. Wang C, Youle RJ. The role of mitochondria in apoptosis. Annu Rev Genet. 2009;43:95-118.

16. Nicholson DW. Caspase structure, proteolytic substrates, and function during apoptotic cell death. Cell Death Differ. 1999;6:10281042.

17. Yacobi K, Wojtowicz A, Tsafriri A, Gross A. Gonadotropins enhance caspase- 3 and -7 activity and apoptosis in the theca-interstitial cells of rat preovulatory follicles in culture. Endocrinology. 2004;145:1943-1951.

18. Oliver FJ, de la Rubia G, Rolli V, Ruiz-Ruiz MC, de Murcia G, Murcia JM. Importance of poly(ADP-ribose) polymerase and its cleavage in apoptosis. Lesson from an uncleavable mutant. J Biol Chem. 1998;273:33533-33539.

19. Johnson GL, Lapadat R. Mitogen-activated protein kinase pathways mediated by ERK, JNK, and p38 protein kinases. Science. 2002; 298:1911-1912.

20. Olson JM, Hallahan AR. p38 MAP kinase: a convergence point in cancer therapy. Trends Mol Med. 2004;10:125-129.

21. Sah JF, Balasubramanian S, Eckert RL, Rorke EA. Epigallocatechin3-gallate inhibits epidermal growth factor receptor signaling pathway. Evidence for direct inhibition of ERK1/2 and AKT kinases. $J$ Biol Chem. 2004;279:12755-12762.

22. Rockwell P, Martinez J, Papa L, Gomes E. Redox regulates COX-2 upregulation and cell death in the neuronal response to cadmium.
Cell Signal. 2004;16:343-353.

23. Himes SR, Sester DP, Ravasi T, Cronau SL, Sasmono T, Hume DA. The JNK are important for development and survival of macrophages. J Immunol. 2006;176:2219-2228.

24. Franklin CC, Srikanth S, Kraft AS. Conditional expression of mitogen-activated protein kinase phosphatase-1, MKP-1, is cytoprotective against UV-induced apoptosis. Proc Natl Acad Sci U S A. 1998;95:3014-3019.

25. Callsen D, Brüne B. Role of mitogen-activated protein kinases in Snitrosoglutathione-induced macrophage apoptosis. Biochemistry. 1999;38:2279-2286.

26. Kim WJ, Lee SJ, Choi YD, Moon SK. Decursin inhibits growth of human bladder and colon cancer cells via apoptosis, G1-phase cell cycle arrest and extracellular signal-regulated kinase activation. Int JMol Med. 2010;25:635-641.

27. Jang J, Jeong SJ, Kwon HY, Jung JH, Sohn EJ, Lee HJ, Kim JH, Kim $\mathrm{SH}, \mathrm{Kim} \mathrm{JH}, \mathrm{Kim} \mathrm{SH}$. Decursin and doxorubicin are in synergy for the induction of apoptosis via STAT3 and/or mTOR pathways in human multiple myeloma cells. Evid Based Complement Alternat Med. 2013;2013:506324.

28. Choi HS, Cho SG, Kim MK, Kim MS, Moon SH, Kim IH, Ko SG. Decursin in Angelica gigas Nakai (AGN) enhances doxorubicin chemosensitivity in NCI/ADR-RES ovarian cancer cells via inhibition of P-glycoprotein expression. Phytother Res. 2016;30:20202026.

29. Kim E, Nam J, Chang W, Zulfugarov IS, Okhlopkova ZM, Olennikov D, Chirikova NK, Kim SW. Angelica gigas Nakai and decursin downregulate Myc expression to promote cell death in B-cell lymphoma. Sci Rep. 2018;8:10590.

30. Palmer AC, Sorger PK. Combination cancer therapy can confer benefit via patient-to-patient variability without drug additivity or synergy. Cell. 2017;171:1678-1691.e13.

31. Westphal M, Lamszus K. The neurobiology of gliomas: from cell biology to the development of therapeutic approaches. Nat ReV Neurosci. 2011;12:495-508.

32. Lee JK, Jeong JW, Jang T, Lee GW, Han H, Kang JS, Kim IH. Decursin attenuates kainic acid-induced seizures in mice. Neuroreport. 2014;25:1243-1249. 